Conclusions This preliminary analysis suggests that CYP1A2 and CYP2C19 polymorphisms may be associated with toxicity in patients with RCC treated with sunitinib. Polymorphisms associated with toxicity and survival in this preliminary analysis are being validated in an independent cohort of 95 RCC patients treated with sunitinib.

No conflict of interest.

\section{PHC-019 PHARMACOGENETICS OF ANTIPLATELET AGENTS: TOWARDS PERSONALISED TREATMENT?}

\section{doi:10.1136/ejhpharm-2013-000276.364}

${ }^{1} \mathrm{C}$ Dávila Fajardo, 2J Sánchez-Ramos, 2P Toledo Frías, 'C García, 'C Gómez, 'C Marín, ${ }^{2} \mathrm{~A}$ Bautista, ${ }^{2} \mathrm{~F}$ Burillo, $1 \mathrm{~J}$ Cabeza Barrera. 'San Cecilio University Hospital, Hospital Pharmacy, Granada, Spain; ${ }^{2}$ San Cecilio University Hospital, Cardiology, Granada, Spain

Background Clopidogrel antiplatelet effects differ according to genotypes $\mathrm{ABCB} 1$ and $\mathrm{CYP} 2 \mathrm{C} 19$, establishing normal, intermediate and slow metabolizers. The intermediate and slow metabolizers and poor transporters are responsible for the poor response to the antiplatelet drug.

Purpose To determine the prevalence of CYP2C19 and ABCB1 genetic polymorphism in the normal Andalusian population (control) and compare it with other populations as a step to implement this determination in clinical practise.

Materials and Methods We genotyped 100 controls from the Andalusian DNA bank for CYP2C19 * 2 (rs4244285), CYP2C19 * 3 (rs4986893) and ABCB1 (rs1045642) using TaqMan probes and allelic discrimination technique. Statistical analysis for allelic and genotypic distributions was calculated by chi-squared test or Fisher's exact test, when necessary, using the Statcalc software packages.

Results Genotype frequencies CYP2C19 $\left({ }^{*} 2\right)$ in the Andalusian population: ${ }^{*} 1 /{ }^{*} 1$ : $73 \%,{ }^{*} 1 /{ }^{*} 2: 25 \%,{ }^{*} 2 /{ }^{*} 2: 2 \%$, and CYP2C19 ${ }^{*} 3$ : none; the same results as in HapMap (NW European ancestry) population. ABCB1: Andalusian population: CC 36\%, CT 44\%, TT 20\%; HapMap population CC $27 \%$, CT 50\%, TT 23\%. Allelic frequencies: NW European ancestry HapMap CYP2C19 * 2: G is $85 \%$ and A is 15\%, the same as our Andalusian control results. ABCB1: HapMap $\mathrm{C}$ allele frequency is $45 \%$ and the $\mathrm{T}$ is $55 \%$, and our frequencies are $57 \%$ C and $43 \%$ T. Having made the genotype study, $59 \%$ of the controls were sensitive to clopidogrel and $41 \%$ resistant to it.

\section{Conclusions}

- Frequencies for CYP2C19*2 and *3 were similar to those reported in other studies. The frequencies for ABCB1 differed slightly

- It is necessary to perform this type of study in patients with acute coronary syndrome undergoing a percutaneous coronary intervention, to ensure effective treatment as it is documented that clopidogrel is not an effective drug in polymorphisms with allele CYP2C19 $* 2\left(* 1 /{ }^{*} 2\right.$ and $\left.{ }^{*} 2 /{ }^{*} 2\right)$ and/or ABCB1 TT.

No conflict of interest.

\section{PHC-020 PHARMACOKINETICALLY GUIDED DOSE ADJUSTMENT OF 5-FLUOROURACIL (5-FU) IN GASTROINTESTINAL CANCER PATIENTS}

doi:10.1136/ejhpharm-2013-000276.365

A Egüés, A Aldaz, M Marin, N Alzueta, L Zufia, A Bermejo. Clinica Universitaria de Navarra, Pharmacy, Pamplona, Spain

Background Appropriate dosing of chemotherapeutic drugs is critical to reducing mortality and increasing progression-free survival. 5-fluorouracil (5-FU) is a widely used chemotherapeutic drug in gastrointestinal cancer. The standard approach to dosing $5-\mathrm{FU}$ is based on body surface area (BSA). However, BSA does not account for many of the factors that are responsible for 5-FU clearance such as age, gender, genotype, disease state, drug-drug interactions, organ dysfunction and co-morbidities. Clinical evidence indicates a strong correlation between both toxicity and therapeutic efficacy and total 5-FU exposure expressed as area under the curve (AUC) concentration. This evidence make 5-FU a good candidate for pharmacokinetic (PK)-guided dosing.

Purpose To evaluate the role of therapeutic drug monitoring (TDM) of 5-FU in daily clinical oncology practise.

Materials and Methods Prospective study of adult patients diagnosed with gastrointestinal cancer treated with infusion schedule regimes based on high doses of 5-FU $\left(2.5-3.2 \mathrm{~g} / \mathrm{m}^{2}\right.$ in $24-46 \mathrm{~h}$ infusion) in a university hospital. All patients were included regardless of disease state or general clinical status. Individual pharmacokinetic parameters were calculated based on anthropometrics and history of 5-FU administration using the Bayesian software programme (USC*Pack). In the first cycle the dose was calculated using the BSA, and subsequent doses were adjusted to an optimal target AUC of 30-35 $\mathrm{mg} \cdot \mathrm{h} / \mathrm{L}$.

Results Fifty-four patients were included in the study. Male/ female ratio was $31 / 23$, and average age and weight were $60.9 \pm 12.8$ years and $72.2 \pm 16.9 \mathrm{Kg}$. Mean estimated pharmacokinetic parameters for volume of distribution and 5 -FU clearance were $0.49 \pm 0.08$ $\mathrm{L} / \mathrm{Kg}$ and $203 \pm 68.6 \mathrm{~L} / \mathrm{h}$, respectively. To achieve the target AUC of $30-35 \mathrm{mg} \cdot \mathrm{h} / \mathrm{L}$, the dose had to be increased in $33(86.8 \%)$ patients and adjusted downward in $5(13 \%)$. No adjustment was needed in 16 patients $(29.6 \%)$. When the estimate was based on BSA, 30 patients $(55.6 \%)$ had AUC $<25 \mathrm{mg} \cdot \mathrm{h} / \mathrm{L}$

Conclusions BSA-based 5-FU dosing approaches are limited when it comes to achieving optimal plasma levels in most patients. Pharmacokinetically guided dosing represents a better strategy to improve the efficacy and safety of 5-FU.

No conflict of interest.

\section{PHC-021 PHARMACOKINETICS OF PIPERACILLIN AND CIPROFLOXACIN IN CRITICALLY ILL PATIENTS UNDERGOING CONTINUOUS VENOVENOUS HAEMODIALYSIS (CVVHD) OR CONTINUOUS VENOVENOUS HAEMODIAFILTRATION (CVVHDF)}

doi:10.1136/ejhpharm-2013-000276.366

F Scheer, I Kraemer. University Medical Center, Pharmacy Department, Mainz, Germany

Background Critically ill patients on intensive care units are often suffering from sepsis and multiorgan failure causing a high mortality rate. In the presence of acute renal failure (ARF) survival can be improved by continuous renal replacement therapy (CRRT). However these procedures are known to be associated with underdosing of the antibiotic agents.

Purpose To investigate the efficacy and safety of antibiotic treatment, especially piperacillin/tazobactam and ciprofloxacin in patients undergoing CRRT.

Materials and Methods A total of 24 patients with ARF treated with CRRT were enrolled in the clinical trial. Plasma and dialysate concentrations of piperacillin/tazobactam and ciprofloxacin were measured in the steady state treatment phase. Serum concentrations of piperacillin and ciprofloxacin were analysed by a validated HPLC method. Optimum exposure to piperacillin is to be expected when serum concentrations are maintained 4-5 times higher than the minimal inhibitory concentration (MIC), i.e. above $64 \mathrm{mg} / \mathrm{l}$ Optimum exposure to ciprofloxacin is given when the ratio (AUIC) of the area under the curve (AUC) and MIC is $\geq 125 \mathrm{~h}$. In addition the Cmax/MIC ratio should amount to $\geq 10$.

Results For 10 of 21 patients treated with piperacillin/tazobactam $4 / 0.5 \mathrm{~g}$ three times a day plasma concentrations lower than $64 \mathrm{mg} / 1$ 